\title{
A note on the converse of the Lefschetz theorem for $G$-maps
}

\author{
by M. IzYDOREK (Gdańsk) and A. VidAL (La Laguna)
}

\begin{abstract}
The purpose of this note is to prove the converse of the Lefschetz fixed point theorem (CLT) together with an equivariant version of the converse of the Lefschetz deformation theorem (CDT) in the category of finite $G$-simplicial complexes, where $G$ is a finite group.
\end{abstract}

1. Introduction. We denote by $G$ a finite group and by $X$ a finite $G$ simplicial complex. Given an equivariant map $f: X \rightarrow X$, for each subgroup $H \leq G$ one can consider its restriction $f^{H}: X^{H} \rightarrow X^{H}$ to the space $X^{H}$ of points fixed by $H$. If $f$ is equivariantly deformable to a fixed point free map then so is $f^{H}$ (in the classical sense). Therefore the Lefschetz number $L\left(f^{H}\right)$ of $f^{H}$ must be zero for any $H \leq G$. One would like to know when the vanishing of $L\left(f^{H}\right), \forall H \leq G$, is a sufficient condition for $f$ to be equivariantly deformable to a fixed point free map.

This problem has been treated for compact smooth $G$-manifolds by D. Wilczyński $[\mathrm{W}]$, A. Vidal [V], L. Borsari and D. Gonçalves [B-G] and recently by E. Fadell and $\mathrm{P}$. Wong $[\mathrm{F}-\mathrm{Wo}]$. In these papers sufficient conditions on a $G$-manifold are given in order to obtain an affirmative answer to the above problem. Our note is a natural extension of these results to the category of finite $G$-simplicial complexes. We use the notion of equivariant Nielsen number for self $G$-maps of $G$-ENR's introduced by P. Wong in [Wo] in the proof of an equivariant version of CLT and CDT.

2. The converse of the Lefschetz fixed point theorem. Let $X$ be a finite $G$-simplicial complex. For any subgroup $H$ in $G$ we denote by $W H$ the Weyl group of $H$. Let $(H)$ denote the conjugacy class of $H$. The set of such classes can be partially ordered in the following way: $(H) \leq(K)$ if $H$

1991 Mathematics Subject Classification: Primary 55M20; Secondary 57S17.

Key words and phrases: equivariant Nielsen number, $G$-simplicial complex, equivariant map, fixed point. 
is subconjugate to $K$, that is, $H$ is conjugate to some subgroup of $K$. Given $x \in X$, the isotropy group $G_{x}$ of $x$ is defined by $G_{x}=\{g \in G: g x=x\}$. An isotropy class $(H)$ is said to be an isotropy type of $X$ provided there is an $x \in X$ such that $\left(G_{x}\right)=(H)$. Let $\left\{\left(H_{i}\right)\right\}, i=1, \ldots, k$, denote the set of all isotropy types of $X$. We can choose the numbering so that $\left(H_{j}\right) \leq\left(H_{i}\right)$ implies $i \leq j$. This induces a filtration of $G$-complexes $X_{0} \subset X_{1} \subset \ldots$ $\ldots \subset X_{k}=X$, where $X_{i}=\left\{x \in X:\left(G_{x}\right)=\left(H_{j}\right), j \leq i\right\}$ and $X_{0}=\emptyset$. Clearly, each equivariant map $f: X \rightarrow X$ preserves the filtration, i.e., $f\left(X_{i}\right) \subset X_{i}, i=0,1, \ldots, k$. We will denote by $f_{i}$ the restriction $f_{\mid X_{i}}$. Associated with $f$ there is a family of $W H_{i}$-equivariant maps $f^{H_{i}}: X^{H_{i}} \rightarrow$ $X^{H_{i}}$, where $X^{H_{i}}=\left\{x \in X: H_{i} \subset G_{x}\right\}$ and $f^{H_{i}}=f_{\mid X^{H_{i}}}$. We say that a connected finite simplicial complex $X$ is of type $S$ if the dimension of $X$ is at least 3 and if for all 0 - and 1 -simplices $\sigma$, the $\operatorname{link} \operatorname{lk}(\sigma, X)$ is connected. A finite $G$-simplicial complex $X$ is said to satisfy the equivariant Shi condition if every connected component of $X^{H}$ is of type $S$ for every isotropy type (H) of $X$.

THEOREM 2.1 (CLT). Let $X$ be a finite $G$-simplicial complex satisfying the equivariant Shi condition such that $X^{H_{i}}$ is simply connected and $X^{H_{i}}$ $X_{i-1}$ is connected for $i=1, \ldots, k$. Then for any $G$-map $f: X \rightarrow X$ with all Lefschetz numbers $L\left(f^{H_{i}}\right)=0$ there is a map $f^{\prime}$ equivariantly homotopic to $f$ which is fixed point free.

Notice that if $G$ acts trivially on $X$ we have the classical theorem (VIII, $\mathrm{D}$, in $[\mathrm{B}])$. The construction of $f^{\prime}$ proceeds by induction on isotropy types on $X$, starting from the maximal type. We will need the following two lemmas.

LemMa 2.2. Let $(Y, A)$ be a finite pair of $G$-simplicial complexes. Let $f: Y \rightarrow Y$ be a $G$-map such that the restriction $f_{\mid A}: A \rightarrow Y$ is equivariantly deformable to a fixed point free map. Then there exist a closed invariant neighborhood $N$ of $A$ and a G-homotopy $S: Y \times I \rightarrow Y$ such that $S_{0}=f$ and $\operatorname{Fix}\left(S_{1}\right) \subset Y-N$.

Pr o of. Let $F: A \times I \rightarrow Y$ be an equivariant homotopy of $f_{\mid A}$ to a fixed point free map. Since the inclusion $A \hookrightarrow Y$ is a $G$-cofibration, $F$ can be extended to a $G$-homotopy $S: Y \times I \rightarrow Y$ with $S_{0}=f$. The assertion of the lemma follows directly from the fact that $\operatorname{Fix}\left(S_{1}\right) \subset Y-A$ is compact.

Lemma 2.3 (Inductive step). Let $(Y, A)$ be a finite pair of $G$-simplicial complexes such that $Y$ is a simply connected space of type $S$ and $Y-A$ is a connected free $G$-space. Let $f: Y \rightarrow Y$ be as in Lemma 2.2. Then $L(f)=0$ implies $f$ is equivariantly deformable (relative to $A$ ) to a fixed point free map. 
Proof. By Lemma 2.2 we can assume that $\operatorname{Fix}(f)$ lies in $Y-N$ for some closed invariant neighborhood $N$ of $A$. Now, for $U=f^{-1}(Y-N)$ we have a map $f_{\mid U}: U \rightarrow Y$ satisfying all the assumptions of Prop. 4.2 of [Wo]. Thus there exist a $G$-simplicial complex $Z \subset U$ with $\operatorname{Fix}(f) \subset$ int $Z$ and a $G$-homotopy $T: U \times I \rightarrow Y$ (relative to $U-\operatorname{int} Z$ ) such that $T_{0}=f_{\mid U}$ and $\operatorname{Fix}\left(T_{1}\right)$ is a finite set. This homotopy can be extended to the whole space by $f$ and the extension will also be denoted by $T$. Notice that $\operatorname{Fix}\left(T_{1 \mid U}\right)=$ $\operatorname{Fix}\left(T_{1}\right)$. Since $Y$ is simply connected $T_{1}$ has exactly one Nielsen fixed point class. Moreover, as $Y-A$ is connected we also have an equivariant Nielsen class and this class is nonessential since $L(f)=0$. Corollary 5.7 of [Wo] completes the proof.

Proof of Theorem 2.1. We proceed by induction on isotropy types on $X$, starting from the maximal type. Suppose that $h: X \rightarrow X$ is a $G$-map equivariantly homotopic to $f$ such that $h_{i-1}: X_{i-1} \rightarrow X_{i-1}$ is fixed point free. Putting $Y=X^{H_{i}}, A=X^{H_{i}} \cap X_{i-1}$ and $G=W H_{i}$ we deduce, by Lemma 2.3 , that $h_{\mid X^{H_{i}}}$ is $W H_{i^{-}}$-homotopic relative to $X^{H_{i}} \cap X_{i-1}$ to a fixed point free map. Denote this $W H_{i}$-homotopy of $h_{\mid X^{H_{i}}}$ by $h_{t}^{H_{i}}$. We extend it to a $G$-homotopy $h_{t}: X_{i} \rightarrow X_{i}$ by the formula $h_{t}(y):=g h_{t}^{H_{i}} g^{-1} y$, $G_{y}=g H_{i} g^{-1}$. Clearly $h_{1}$ is fixed point free. Since $X_{i} \hookrightarrow X$ is a $G$-cofibration the map $\varphi: X \cup X_{i} \times I \rightarrow X$ given by $\varphi(x, 0)=h(x), \varphi(x, t)=h_{t}(x)$ can be equivariantly extended to a map $\Phi: X \times I \rightarrow X$. Thus we obtain a map $\Phi_{1}: X \rightarrow X G$-homotopic to $f$ and fixed point free on $X_{i}$.

The same proof remains valid for

Corollary 2.4. Let $X$ be a finite $G$-simplicial complex satisfying the equivariant Shi condition such that $X^{H_{i}}-X_{i-1}$ is connected and the homomorphism $i_{*}: \pi_{1}\left(X^{H_{i}}-X_{i-1}\right) \rightarrow \pi_{1}\left(X^{H_{i}}\right)$ induced by inclusion is epimorphic for $i=1, \ldots, k$. Let $f: X \rightarrow X$ be a $G$-map with all Nielsen numbers $N\left(f^{H_{i}}\right)=0$. Then $f$ is equivariantly deformable to a fixed point free map.

3. The converse of the Lefschetz deformation theorem. In this section we prove a theorem for the identity map similar to Theorem 2.1 under weaker assumptions, not demanding that $X^{H_{i}}$ is simply connected for $i=1, \ldots, k$.

THEOREM 3.1 (CDT). Let $X$ be a finite $G$-simplicial complex satisfying the equivariant Shi condition. If for each $i=1, \ldots, k$, the Euler characteristic $\chi\left(X^{H_{i}}\right)=0$ and $X^{H_{i}}-X_{i-1}$ is connected then the identity $1_{X}$ is equivariantly deformable to a fixed point free map.

As in Section 2 we need two lemmas. However, the fact that we do not assume simple connectedness of $X^{H_{i}}$ forced us to be more careful with the construction of an appropriate deformation of $1_{X}$ in the inductive step. 
Let $N \subset X$ be a $G$-subcomplex of $X$. We denote by $N^{*}$ the $G$-subcomplex of $X$ consisting of all simplices $\sigma$ of $X$ such that $\bar{\sigma} \cap N=\emptyset$. We call it the complement of $N$ in $X$.

Lemma 3.2. Let $(Y, A)$ be a finite pair of $G$-simplicial complexes. Suppose that the restriction of $1_{X}$ to $A$ is equivariantly deformable to a fixed point free map. Then there exist a closed regular neighborhood $N$ of $A$ and a $G$-homotopy $S: Y \times I \rightarrow Y$ such that $S_{0}=1_{Y}$ and $\operatorname{Fix}\left(S_{1}\right)=N^{*}$.

Proof. Let $F: A \times I \rightarrow A$ be a $G$-homotopy of $1_{A}$ with $\operatorname{Fix}\left(F_{1}\right)=\emptyset$. By Theorem 9.9 of $[\mathrm{E}-\mathrm{S}], A$ is a strong deformation retract of its second regular neighborhood $N$. Let $H: N \times I \rightarrow N$ be a homotopy relative to $A$ with $H_{0}=1_{N}$ and $H_{1}=r$ a retraction. Clearly, $N$ is a $G$-subcomplex of $Y$ and we can choose $H$ to be an equivariant homotopy. Let $\varrho: N \rightarrow I$ be a continuous invariant function such that $\varrho^{-1}(0)=\partial N$, the boundary of $N$, and $\varrho^{-1}(1)=A$. Then the map $P: N \times I \rightarrow N$ defined by $P(x, t)=$ $F(r(x), t \varrho(x))$ is a $G$-deformation of $P_{0}=r$. Consequently, we can define an equivariant homotopy $Q: N \times I \rightarrow N$ of the identity $1_{N}$ to a fixed point free map by the formula

$$
Q(x, t)= \begin{cases}H(x, 2 t), & 0 \leq t \leq 1 / 2 \\ P(x, 2 t-1), & 1 / 2 \leq t \leq 1\end{cases}
$$

In particular, $Q(x, 1)$ coincides with the retraction $r$ on the boundary $\partial N$. Let $N^{*}$ be the complement of $N$ in $Y$. For any $x \in Y-\left(N \cup N^{*}\right)$ there is a unique simplex $\tau$ of $Y$ such that $x \in \operatorname{int} \tau$. We put $\sigma_{1}=\bar{\tau} \cap N^{*}$ and $\sigma_{2}=\bar{\tau} \cap N$. Since $\bar{\tau}$ is the join of $\bar{\sigma}_{1}$ and $\bar{\sigma}_{2}, \bar{\tau}=\bar{\sigma}_{1} * \bar{\sigma}_{2}$, there are unique points $p_{1} \in \sigma_{1}, p_{2} \in \sigma_{2}$ and $s \in(0,1)$ such that $x=(1-s) p_{1}+s p_{2}$. Using this notation we define a map $R:\left(Y-\left(\operatorname{int} N \cup \operatorname{int} N^{*}\right)\right) \times I \rightarrow Y$ by

$$
R(x, t)= \begin{cases}(1-s(1+t)) p_{1}+(1+t) s p_{2}, & \frac{1}{t+1} \geq s, \\ Q\left(p_{2},(1+t) s-1\right), & \frac{1}{t+1} \leq s .\end{cases}
$$

Notice that $R(x, 1)=x$ only if $x \in \partial N^{*}$. Finally, a homotopy $S: Y \times I \rightarrow Y$ satisfying the assertion of the lemma can be defined as follows:

$$
S(x, t)= \begin{cases}Q(x, t), & (x, t) \in N \times I, \\ R(x, t), & (x, t) \in\left(Y-\left(\operatorname{int} N \cup \operatorname{int} N^{*}\right)\right) \times I, \\ x, & (x, t) \in N^{*} \times I .\end{cases}
$$

Re mark 3.3. The proof above yields more, namely that for every $\varepsilon>0$ the $G$-homotopy $S$ can be additionally made an $\varepsilon$-homotopy.

LEMMA 3.4. Let $(Y, A)$ be a finite pair of $G$-simplicial complexes such that $Y-A$ is a connected free $G$-space, $Y$ is of type $S$ and has Euler characteristic 
zero. Then the map $f=S_{1}$ as in Lemma 3.2 is G-homotopic to a fixed point free map.

Proof. Put $U=f^{-1}(Y-N)$; then $U$ is a free open invariant neighborhood of $N^{*}$. Let $l$ be the Lebesgue number of an open star covering $\Theta$ of $Y$. By Proposition 4.2 of [Wo] there exist a finite $G$-simplicial complex $Z \subset U$ with $N^{*} \subset$ int $Z$ and an equivariant $\eta$-homotopy $T: U \times I \rightarrow Y$ (relative to $U-\operatorname{int} Z)$ such that $T_{0}=f_{\mid U}, \operatorname{Fix}\left(T_{1}\right)$ is a finite set and $\eta<\frac{1}{2} l$. Clearly, $T$ can be extended to the whole space by $f$ and this extension will also be denoted by $T$. Notice that we still have $\operatorname{Fix}\left(T_{1}\right)=\operatorname{Fix}\left(T_{1 \mid U}\right)$.

We want to show that all fixed points of $T_{1}$ are in the same Nielsen class. Let $z_{1}, z_{2} \in \operatorname{Fix}\left(T_{1}\right)$ and let $\alpha$ be a path from $z_{1}$ to $z_{2}$. Using the homotopy $V: Y \times I \rightarrow Y$ given by

$$
V(x, t)= \begin{cases}S(x, 2 t), & 0 \leq t \leq 1 / 2 \\ T(x, 2 t-1), & 1 / 2 \leq t \leq 1\end{cases}
$$

where $S$ is as in Lemma 3.2, we find that $V(\alpha(s), 0)=\alpha(s)$ is homotopic to $\left(T_{1} \circ \alpha\right)(s)=V(\alpha(s), 1)$ but the homotopy is not relative to endpoints. During the homotopy $z_{1}$ and $z_{2}$ make loops $V\left(z_{1}, t\right), V\left(z_{2}, t\right)$ with diameter not greater than $2 \eta<l$. Thus there are open stars $S t_{1}, S t_{2} \in \Theta$ including $V\left(z_{1}, t\right)$ and $V\left(z_{2}, t\right)$ respectively. Hence, both loops are homotopically trivial (relative to endpoints). Consequently, we have

$$
\alpha \simeq V\left(z_{1}, \cdot\right) * T_{1} \circ \alpha * V\left(z_{2}, \cdot\right)^{-1} \text { rel. }\left\{z_{1}, z_{2}\right\}
$$

and by the contractibility of the loops,

$$
V\left(z_{1}, \cdot\right) * T_{1} \circ \alpha * V\left(z_{2}, \cdot\right)^{-1} \simeq T_{1} \circ \alpha \text { rel. }\left\{z_{1}, z_{2}\right\} .
$$

Since $Y-A$ is connected $\alpha$ can be chosen to avoid $A$ and therefore there is also exactly one equivariant Nielsen class. Now, $\chi(Y)=0$ and this class is nonessential. But $Y$ is of type $S$ and by Corollary 5.7 of [Wo] we have the fixed point free map we are looking for.

Proof of Theorem 3.1. It is the same as that of Theorem 2.1. One only has to replace Lemmas 2.2 and 2.3 by Lemmas 3.2 and 3.4.

R e m a r k 3.5. Notice that the assumption of $X^{H_{i}}-X_{i-1}$ being connected implies that all connected components of $X^{H_{i}}$ have Euler characteristic zero. Clearly the condition $\chi\left(X^{H_{i}}\right)=0$ is not sufficient to deform the identity equivariantly to a fixed point free map.

EXAMPLE 1. Let $M_{1}$ be the boundary-connected sum of two copies of the Cartesian product $D^{4} \times S^{1}$ of a four-dimensional disc and a one-dimensional sphere. For $x_{0} \in \operatorname{int} M_{1}$ take a sufficiently small open disc $\stackrel{\circ}{D}\left(x_{0}\right)$ centered at $x_{0}$ and put $M=M_{1}-\stackrel{\circ}{D}\left(x_{0}\right)$. The boundary $\partial M$ of $M$ is a disjoint union of $S^{4}$ with the connected sum $S^{3} \times S^{1} \# S^{3} \times S^{1}$. 
Consider the manifold $N=M \sqcup_{\partial M} M$ with the natural $\mathbb{Z}_{2}$-action. Then $N^{\mathbb{Z}_{2}}=S^{4} \sqcup S^{3} \times S^{1} \# S^{3} \times S^{1}$ and $\chi(N)=\chi\left(N^{\mathbb{Z}_{2}}\right)=0$, but $1_{N}$ is not equivariantly deformable to a fixed point free map. Notice that in this example neither $N^{\mathbb{Z}_{2}}$ nor $N-N^{\mathbb{Z}_{2}}$ is connected. By a slight modification we can arrange things so that only one of them is not connected. Indeed, let $W$ be the 3 -dimensional real representation of $\mathbb{Z}_{2}=\{1,-1\}$ given by $(-1)(x, y, z)=(-x,-y, z)$, and let $S(W)$ denote the unit sphere. Then for the $\mathbb{Z}_{2}$-manifold $\tilde{N}=N \times S(W)$ we have $\chi(\tilde{N})=\chi\left(\widetilde{N}^{\mathbb{Z}_{2}}\right)=0$ and $\widetilde{N}-\widetilde{N}^{\mathbb{Z}} 2$ is connected.

However, the connectedness of $X^{H_{i}}-X_{i-1}$ is not necessary to deform the identity equivariantly to a fixed point free map, as shown in

EXAMPLE 2 . Let $W_{1}$ be the 2-dimensional representation of $\mathbb{Z}_{2}$ given by $(-1)(x, y)=(-x, y)$, and let $S\left(W_{1}\right)$ be the unit sphere. Consider the torus $T=S\left(W_{1}\right) \times S^{1}$ with the diagonal action of $\mathbb{Z}_{2}$, where $\mathbb{Z}_{2}$ acts trivially on $S^{1}$. Then neither $T^{\mathbb{Z}_{2}}=S^{1} \sqcup S^{1}$ nor $T-T^{\mathbb{Z}_{2}}$ is connected. Nevertheless, the map $f: T \rightarrow T$ defined by $f\left(z_{1}, z_{2}\right)=\left(z_{1},-z_{2}\right)$ is fixed point free and $\mathbb{Z}_{2}$-homotopic to $1_{T}$.

Problem 3.6. Let $X$ be a $G$-complex satisfying the equivariant Shi condition. Assume that all connected components of $X^{H}$ have Euler characteristic zero for any $H \leq G$. Is it true that the identity map $1_{X}$ is equivariantly deformable to a fixed point free map?

We can give an affirmative answer to this question if $X$ is a closed smooth $G$-manifold $M$ and $G$ an abelian group of odd order. If, in addition, we suppose that the dimension of $M$ is odd, then the identity is always equivariantly deformable to a fixed point free map. This is due to the fact that each connected component of $M^{H}$ has even codimension in $M$ and, consequently, all the above-mentioned components have Euler characteristic zero (cf. [Bo]). We believe that the above problem is interesting even for the particular case of a $\mathbb{Z}_{2}$-manifold.

Acknowledgements. This paper was written during a stay at the Centre de Recerca Matemàtica of the Institut d'Estudis Catalans. The authors wish to express their gratitute to Prof. Manuel Castellet for his support and hospitality.

\section{References}

[B-G] L. Borsari and D. Gonçalves, G-deformations to fixed point free maps via obstruction theory, preprint.

[Bo] C. Bowszyc, On the components of the principal part of a manifold with a finite group action, Fund. Math. 115 (1983), 229-233. 
[B] R. Brown, The Lefschetz Fixed Point Theorem, Scott and Foresman, 1971.

[E-S] S. Eilenberg and N. Steenrod, Foundations of Algebraic Topology, Princeton Univ. Press, 1952.

[F-Wo] E. Fadell and P. Wong, On deforming G-maps to be fixed point free, Pacific J. Math. 132 (1988), 277-281.

[V] A. Vidal, On equivariant deformation of maps, Publ. Mat. 32 (1988), 115-121.

[W] D. Wilczyński, Fixed point free equivariant homotopy classes, Fund. Math. 123 (1984), 47-60.

[Wo] P. Wong, Equivariant Nielsen fixed point theory for G-maps, Pacific J. Math. 150 (1991), 179-200.

TECHNICAL UNIVERSITY OF GDAŃSK

DEPARTMENT OF APPLIED MATHEMATICS 80-952 GDAŃSK

POLAND
UNIVERSIDAD DE LA LAGUNA FACULTAD DE MATEMÁTICAS LA LAGUNA, TENERIFE

Reçu par la Rédaction le 28.8.1992 